\title{
Structure-properties correlations unlocked through exquisitely sensitive powder diffraction
}

\author{
P. Khalifah ${ }^{1,2}$ \\ ${ }^{1}$ Stony Brook University, Department of Chemistry, ${ }^{2}$ Brookhaven National Laboratory, Department of Chemistry \\ kpete@bnl.gov
}

The overarching goal of this work is to understand and overcome the performance limitations of industrially relevant battery materials using powder diffraction studies, both through ex situ studies of materials and operando studies of cycling battery cells. However, the normal modalities for the collection and analysis of powder diffraction data typically lack the sensitivity to resolve the structure of battery materials with sufficiently low uncertainty to effectively resolve structure-properties correlations. We have therefore actively been developing new approaches to data collection and analysis that overcome these limitations, permitting us to obtain robust structure-properties correlations for industrially relevant cathode materials and for industrially relevant battery cell designs.

We have recently developed a novel perspective for systematically exploring occupancy defects which we have applied to the study of the important family of NMC battery cathode materials [1]. Using these $f^{*}$ diagrams, we have demonstrated sufficient sensitivity to site occupancies to resolve problems with the conventional atomic form factors used for X-ray diffraction - an error of about 3\% in the case of oxygen. After correcting for these problems and robustly determining atomic displacement parameters, we have demonstrated the ability to unambiguously resolve the nature of key defects as well as to determine defect concentrations with an unprecedented sensitivity of $\sim 0.1 \%$ (absolute), as judged by the agreement between independent refinements of synchrotron and neutron powder diffraction data. From the refined occupancies for a series of NMC compounds, it was possible to determine the energy associated with the formation of anti-site defects, and to conclusively demonstrate that the conventionally accepted mechanism for defect formation was incorrect [2]. Additionally, we have utilized rapid synchrotron powder diffraction methods to carry out multidimensional diffraction studies with fine resolution not just in time but in space as well. In this manner, it has been possible to resolve both vertical [3] and lateral [4] inhomogeneity in battery cells with a sensitivity to the local state of charge (SOC) of $\sim 0.1 \%$. The former has illuminated the performance limitations of exceptionally thick battery cathodes with very high energy densities, while the latter has allowed us to distinguish between different potential failure mechanisms.
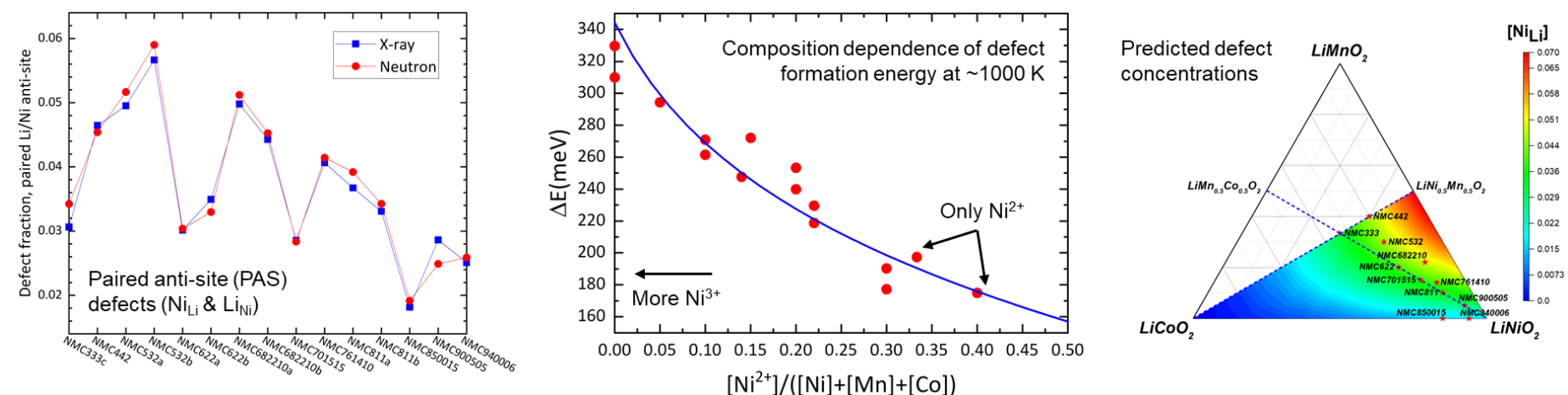

Figure 1. Left: Comparison of paired anti-site defect concentration obtained independently from synchrotron (blue) and neutron (red) powder diffraction data. Center: Defect formation energies calculated based on refined defect concentrations show that the defect formation energy is not constant, but instead varies systematically with the concentration of Ni2+. From the fit to this data, the defect concentration for any NMC composition can be predicted from its composition alone with an uncertainty less than prior experiments.

[1] L. Yin, G. Mattei, Z. Li, J. Zheng, W. Zhao, F. Omenya, C. Fang, W. Li, J. Li, Q. Xie, J.-G. Zhang, M.S. Whittingham, Y.S. Meng, A. Manthiram and P. Khalifah (2018). Rev. Sci. Instrum. 89, 093002.

[2] L. Yin, Z. Li, G. Mattei, J. Zheng, W. Zhao, F. Omenya, C. Fang, W. Li, J. Li, Q. Xie, E. Erickson, J.-G. Zhang, M.S. Whittingham, Y.S. Meng, A. Manthiram, and P. Khalifah (2019). Chem. Mater., 32, 1002-1010.

[3] Z. Li, L. Yin, G. Mattei, M. Cosby, B.-S. Lee, Z. Wu, S.-M. Bak, K. Chapman, X.-Q. Yang, P. Liu, and P. Khalifah (2020). Chem. Mater., 32, 6358.

[4] G. Mattei, Z. Li, A. Corrao, C. Niu, Y. Zhang, B.-Y. Liaw, C. Dickerson, J. Xiao, E. Dufek, and P. Khalifah (2021). Chem. Mater., 33 , 2378.

Keywords: Batteries; synchrotron powder diffraction; neutron powder diffraction; anti-site defects; inhomogeneity

Work was supported by the U.S. Department of Energy as part of the Battery500 Consortium. 